\title{
On instantaneous propagation of low-frequency signals in short transmission lines
}

This paper was downloaded from TechRxiv (https://www.techrxiv.org).

\section{LICENSE}

CC BY 4.0

SUBMISSION DATE / POSTED DATE

20-09-2021 / 23-09-2021

CITATION

Kühn, Steffen (2021): On instantaneous propagation of low-frequency signals in short transmission lines. TechRxiv. Preprint. https://doi.org/10.36227/techrxiv.16645792.v1

DOI

10.36227/techrxiv.16645792.v1 


\title{
On instantaneous propagation of low-frequency signals in short transmission lines
}

\author{
Steffen Kühn \\ September 20, 2021
}

\begin{abstract}
One of the basic assumptions of physics is that information cannot be transferred more rapidly than light. If one equates information with data, then information transfer is a process in which data are transmitted between two remote locations. According to this basic principle, the transmission process should require an appropriate amount of time so that the distance between these locations divided by the needed time does not exceed the speed of light in a vacuum. However, it is technologically feasible to transmit a voice message, music, or any bit sequence at a low bit rate between locations separated by hundreds of meters, with almost no loss of time. Herein, we demonstrate that this behavior most likely does not contradict special relativity, and we explain this phenomenon based on a model of a superconducting cable.
\end{abstract}

\section{Contents}

\section{Introduction}

II Model of superconducting cable

\section{Propagation of low-frequency signals}

IV Solution of the ostensible paradox

V Conclusions

\section{References}

\section{INTRODUCTION}

The statement that information propagation at superluminal speeds is impossible is usually interpreted as follows: When a data set is transferred, a certain time must elapse before the first data bit can reach the receiver, and this time can never be smaller than the spatial distance between sender and receiver divided by the speed of light in a vacuum. However, this interpretation is wrong and contradicts actual findings.

One can easily establish an experimental setup to demonstrate that this interpretation is incorrect. Ideally, superconducting cables would be used for this setup. If such a superconducting cable were arranged in a circle so that the ends were almost touching and then a low-frequency signal of any form were applied to the cable at one end of the line, it would be found that the output signal is always perfectly synchronous with the signal at the input, with no delay at all. To the best of the author's knowledge, measurements of this type have not yet been performed with a superconductor; however simulations with LTspice show exactly the predicted effect. Furthermore, measurements with ordinary Cat 5 or coaxial cables also confirm the effect, whereby delays result from the non-zero resistance, which are nevertheless too small for subluminal signals. These measurement results are in excellent agreement with simulation results as well (see references in [3]).

For this phenomenon to be observed, two conditions must be met. First, the line must not be terminated with a resistor; rather, it has to be decoupled with a very high impedance. Second, the input signal should be sufficiently band-limited, i.e., it must not contain any frequency components higher than a certain cut-off frequency, which depends on the cable length.

At first glance, this effect seems to contradict the special theory of relativity. However, the analysis presented in this article shows that this is probably not the case. Rather, the statement that information propagation at superluminal speeds is impossible is misleading and overly simplistic.

\section{MOdEL OF SUPERCONDUCTING CABLE}

The transfer function for a cable of length $l$ at location $x \in$ $[0 \ldots l]$ without resistance and without termination is

$$
H(x, \omega)=\frac{\cos \left(\frac{\omega}{c}(l-x)\right)}{\cos \left(\frac{\omega}{c} l\right)},
$$

where $c=1 / \sqrt{L^{\prime} C^{\prime}}$ is the wave velocity [3]. The parameters $L^{\prime}$ and $C^{\prime}$ represent the inductance and capacitance per meter of cable length. Note that in this model, the wave velocity does not depend on the angular frequency $\omega$ and is therefore the same for all frequencies. As far as we know, in real cables, the product of $L^{\prime}$ and $C^{\prime}$ always has a value such that the wave velocity $c$ does not exceed the speed of light in a vacuum $c_{0}$.

Because there is no termination, a pulse $\delta(t)$ given to the line would, after running to the end, reflect at the end of the line, travel back to the initial position, and then be reflected again. This process would then continue to repeat. Thus, the output signal $h(t, x)$, which would be measured with high-impedance decoupling at location $x$, would be

$$
\begin{array}{r}
h(x, t)=\sum_{k=0}^{\infty}(-1)^{k}\left(\delta\left(t-\frac{l}{c}\left((2 k+1)-\frac{l-x}{l}\right)\right)+\right. \\
\left.\delta\left(t-\frac{l}{c}\left((2 k+1)+\frac{l-x}{l}\right)\right)\right)
\end{array}
$$

and would represent the impulse response of the cable. We can verify that the output signal is indeed the impulse response of 
the superconducting transmission line by substituting equations (1) and (2) into the integral of the Fourier transform:

$$
H(x, \omega)=\int_{-\infty}^{+\infty} h(x, t) e^{-\mathrm{i} \omega t} \mathrm{~d} t .
$$

Note that if the cable were not superconducting, an additional loss factor would appear in the impulse response (2). This term would complicate the calculations but would not fundamentally change the discussion.

Up to this point, everything is as expected. For example, if one performs a measurement at the end of the line, i.e., at $x=l$, the impulse response simplifies to

$$
h(l, t)=2 \sum_{k=0}^{\infty}(-1)^{k} \delta\left(t-(2 k+1) \frac{l}{c}\right),
$$

and it becomes obvious that the first impulse reaches the end of the line for $k=0$ at time $t=\frac{l}{c}$. All succeeding reflections for $k>0$ reach the end of the line even later. As one can clearly see, transmission of a Dirac impulse in the transmission line is possible only at velocity $c$ which is lower than the speed of light in a vacuum $c_{0}$ for typical values of $L^{\prime}$ and $C^{\prime}$.

\section{Propagation of LOW-FreQuency Signals}

If an arbitrary input signal $s_{i}(t)$ is applied, instead of an impulse $\delta(t)$, to the transmission line, the output signal $s_{o}(x, t)$ at location $x$ at time $t$ is given by the convolution of the impulse response and input signal, i.e.

$$
s_{o}(x, t)=h(x, t) * s_{i}(t)=\int_{-\infty}^{+\infty} h(x, t-\tau) s_{i}(\tau) \mathrm{d} \tau .
$$

The Fourier transform of the output signal is

$$
S_{o}(x, \omega)=\int_{-\infty}^{+\infty} s_{o}(x, t) e^{-\mathrm{i} \omega t} \mathrm{~d} t .
$$

Inserting equation (5) gives

$$
\begin{aligned}
S_{o}(x, \omega) & =\int_{-\infty}^{+\infty}\left(\int_{-\infty}^{+\infty} h(x, t-\tau) e^{-\mathrm{i} \omega t} \mathrm{~d} t\right) s_{i}(\tau) \mathrm{d} \tau \\
& =\left(\int_{-\infty}^{+\infty} h(x, t) e^{-\mathrm{i} \omega t} \mathrm{~d} t\right)\left(\int_{-\infty}^{+\infty} s_{i}(\tau) e^{-\mathrm{i} \omega \tau} \mathrm{d} \tau\right) \\
& =H(x, \omega) S_{i}(\omega),
\end{aligned}
$$

where $S_{i}(\omega)$ is the Fourier transform of the input signal $s_{i}(t)$.

In practice, signals are often band-limited, which means that they contain negligible levels of signal components with frequencies above a certain cut-off frequency $f_{\text {max }}$. In this case, only the range of the transfer function (1) for which the condition $|\omega| \leq 2 \pi f_{\text {max }}$ is satisfied is relevant because, the product $H(x, \omega) S_{i}(\omega)$ in equation (7) for angular frequencies $\omega$ above the cut-off frequency is zero in this case.
However, for small angular frequencies $\omega$, the transfer function (1) equals unity as a good approximation and equation (7) becomes

$$
S_{o}(x, \omega) \approx S_{i}(\omega)
$$

Thus, signals that mainly contain frequencies below the cut-off frequency

$$
f_{\text {max }} \approx \frac{c}{32 l}
$$

can apparently be transmitted without any time delay and with almost no distortion. In this case, the following equation applies:

$$
s_{o}(x, t) \approx s_{i}(t) .
$$

Consequently, the input signal appears to be nearly unchanged and seemingly instantaneous at the output $x=l$ of the line.

As an example, an audio signal contains no relevant information above $f_{\text {max }}=15 \mathrm{kHz}$. Using equation (9), it follows that such a signal can be transmitted in a superconductor with a length of up to $l \approx 625 \mathrm{~m}$ with practically no changes or delays.

Another example is a random bit sequence with a bit rate of $5 \mathrm{kBit} / \mathrm{s}$. This bit rate is so low that there is a time gap of $200 \mu \mathrm{s}$ between the transmission of two bits. These bits can also be interpreted as samples of a continuous-time signal generated by sampling with a sampling rate of $5 \mathrm{kHz}$. This continuoustime signal must be band-limited to $10 \mathrm{kHz}$ according to the Nyquist-Shannon sampling theorem. Consequently, the bit sequence can be converted back into a band-limited continuoustime signal via the Whittaker-Shannon interpolation, which can then be transmitted without time loss for a cable length of up to $900 \mathrm{~m}$.

If one performs corresponding measurements, the result

$$
\text { input signal }=\text { output signal }
$$

would appear very surprising and paradoxical for someone who follows conventional thinking, as the transmitter and receiver are several hundred meters apart from each other. However, as shown in section II, the wave velocity $c$, as well as all phase velocities, is not higher than the speed of light in a vacuum $c_{0}$. The effect is therefore somewhat paradoxical.

\section{Solution of the ostensible Paradox}

The paradox is resolved if a pure sine wave of the form

$$
s_{i}(t)=\sin \left(\omega_{s} t\right)
$$

is used as an input signal. The output signal $s_{o}(x, t)$ can then be calculated by convolution of the input signal (11) with the impulse response (2) according to equation (5). In this particular case, we obtain the output signal:

$$
\begin{aligned}
s_{o}(x, t)=\sum_{k=0}^{\infty}(-1)^{k} & \left(\sin \left(\omega_{s}\left(t-\frac{x}{c}-\frac{2 k l}{c}\right)\right)+\right. \\
& \left.\sin \left(\omega_{s}\left(t+\frac{x}{c}-\frac{2(k+1) l}{c}\right)\right)\right)
\end{aligned}
$$

after rearranging the terms. It can be noted that running waves with phase velocity $c$ are present in the transmission line, 
with one wave traveling to the left and the other to the right. Together, the two running waves form a standing wave, and there appears to be no motion.

Incidentally, the infinite sum in equation (12) can be further simplified, and we find that

$$
s_{o}(x, t)=\frac{\cos \left(\omega_{s} \frac{l-x}{c}\right)}{\cos \left(\omega_{s} \frac{l}{c}\right)} \sin \left(\omega_{s} t\right) .
$$

For low angular frequencies $\omega_{s}$ or for a small line length $l$, we then obtain the approximation

$$
s_{o}(x, t) \approx \sin \left(\omega_{s} t\right) .
$$

If we were to incorrectly assume that there is only one running wave in the cable, which moves from the input to the output, we would choose the following approach:

$$
\hat{s}_{o}(x, t)=\sin \left(\omega_{s}\left(t-\frac{x}{v_{p}\left(\omega_{s}\right)}\right)\right) .
$$

However, this approach would only be compatible with the measurement result (14) if we assume a phase velocity $v_{p}\left(\omega_{s}\right)$ that is infinite. It should be mentioned that in cables with a non-vanishing resistance, a running wave can indeed be observed for which the apparent phase velocity is not infinite, but may be significantly faster than the speed of light in a vacuum $c_{0}$.

In conclusion, the apparent paradox is resolved by recognizing the presence of a standing wave in the cable and by realizing that it is wrong to assume a running wave as in equation (15). However, it is also clear that it is technically possible to instantaneously transfer data sets between two distant points in space. The key point, however, is that the information in the data sets must exist for a sufficiently long time before being sent.

If a random process generates a single bit every $200 \mu \mathrm{s}$ in a completely unpredictable manner, each bit would first have to be input as an impulse to a low-pass filter, which performs a Whittaker-Shannon interpolation. The output can be transmitted in the transmission line. The transfer is then instantaneous, but the Whittaker-Shannon interpolation requires time. If this time is taken into account, we find that the information generated by the random process cannot be transmitted faster than the wave velocity $c$.

However, if a physical or technical process is already lowfrequency, then it is possible to observe and monitor this process remotely without any time delay, based on the effect described in this article. For example, it would be possible to transmit the positions of objects to a remote location without any loss of time, as massive physical objects cannot change their position abruptly. As a general rule, it can be argued that any band-limited physical process can be observed quasi-instantaneously if the distance is smaller than a certain minimum. This argument likely applies to all force effects exerted by one object on another, as this is also an inherently low-frequency information transfer [2].

\section{Conclusions}

The article brings us to several interesting conclusions:

(i) The frequently found statement „No information transmission can occur at superluminal speeds" is too simplistic and contradicts experimental findings. In fact, data sets can be transmitted instantaneously if the signal to be transmitted has a sufficiently low frequency and the distance between the locations is not too large.

(ii) Instantaneous data transmission does not require phase or wave velocities higher than the speed of light in a vacuum $c_{0}$. Instead, one can exploit standing waves. This form of instantaneous data transmission is most likely not in conflict with the special theory of relativity because an unpredictable trigger event cannot be transmitted faster than at the wave velocity $c$.

(iii) When measuring phase velocities, care must be taken to ensure that the running wave is moving in only one direction and that there is no wave at the same frequency moving in the opposite direction. If this possibility is neglected, one may conclude that superluminal phase velocities occur. However, superluminal one-way phase velocities contradict the special theory of relativity, because this would allow superluminal data transmission over arbitrarily long distances [4].

(iv) The described effect may explain the experimental observation that forces, such as the Coulomb force, seem to propagate instantaneously at short distances [5], [1].

(v) Further assessments should be conducted to determine whether the wave effect studied here plays a role in quantum teleportation and in quantum eraser experiments.

\section{REFERENCES}

[1] R. de Sangro, G. Finocchiaro, P. Patteri, M. Piccolo, and G. Pizzella. Measuring propagation speed of coulomb fields. The European Physical Journal C, 2015

[2] Wolfgang Engelhardt. Relativity of time and instantaneous interaction of charged particles. American Journal of Modern Physics, 2015.

[3] Steffen Kühn. General analytic solution of the telegrapher's equations and the resulting consequences for electrically short transmission lines. Journal of Electromagnetic Analysis and Applications, 12:71-87, 01 2020.

[4] Steffen Kühn. Inadequacies of Sommerfeld's front velocity definition. In review, 2021.

[5] W. D. Walker. Experimental evidence of near-field superluminally propagating electromagnetic fields. 2000. 